\title{
Nanostructured and functionalized cantilever for sensing organophosphorous compounds
}

\author{
Urelle Biapo $^{l}$, Alessio Ghisolfi ${ }^{1}$, Geoffrey Gerer ${ }^{1,2}$, Denis Spitzer ${ }^{2}$, Valérie Keller ${ }^{l}$, Thomas Cottineau ${ }^{1}$ \\ 1 Institute of Chemistry and Processes for Energy Environment and Health (ICPEES), CNRS-University of Strasbourg \\ UMR 7515, Strasbourg, France \\ 2 Nanomatériaux pour les Systèmes Sous Sollicitations Extrêmes (NS3E), ISL-CNRS-UNISTRA UMR 3208, French- \\ German Research Institute of Saint-Louis, Saint-Louis, France \\ Email : biapofankep@etu.unistra.fr, cottineau@unistra.fr
}

\begin{abstract}
Vertically aligned $\mathrm{TiO}_{2}$ nanorods have been successfully synthesized on the micrometric surface of a cantilever by a two-step procedure involving solvothermal method. Adjusting $\mathrm{TiO}_{2}$ synthesis conditions, first optimized on centimetric silicon substrate, allowed to well-control the morphology of the nanorods, aimed to increase the collection surface of this micromechanical sensor. By using the nanostructured microcantilever for the detection of organophosphorous agent (OPs), a frequency shift of $833 \mathrm{~Hz}$ corresponding to a remarkable enhancement of the sensor sensitivity by a factor of 75 compared to a pristine cantilever is achieved. Moreover, functionalization of this nanostructured cantilever with a tailored functional oxime molecule having strong affinity with OPs compounds significantly improved the performance of the sensor in vapor phase. A frequency shift of $1319 \mathrm{~Hz}$ is obtained at $500 \mathrm{ppm}$ of DMMP with the functionalized sensor.
\end{abstract}

Keywords - $\mathrm{TiO}_{2}$ nanostructure, Organophosphorous agents Micromechanical sensor, Functionalization, Oxime molecule.

\section{INTRODUCTION}

The use of microcantilevers as chemical sensors has demonstrated promising results on various applications including medical and defense fields [1]-[3]. While in biology microcantilevers can be used to follow the hybridization of DNA molecules[4], in the field of chemistry this technology can be employed to control the presence of moisture [5], volatile organic compounds [6],[7], or others specific target in environment [8],[9]. Thanks to an actuation system, the cantilever oscillates at its natural resonant frequency, which is directly related to the spring constant and to the mass of the beam. Any change of the mass of the cantilever, for instance due to adsorption of molecules will result in a variation of the resonance frequency $\left(f_{0}\right)$. In this study, we employed this type of micromechanical sensor for the chemical detection of organophosphorous compounds (OPs) taking advantage of its gravimetric sensor properties. The OPs molecules used as chemical warfare agents (Sarin or Soman) but also as pesticides (Glyphosate) are highly toxics and are considered as one of the most dangerous threats for animals and humans, because they are effective at very low concentration [10]. In order to improve the detection of these compounds, we propose a novel strategy to improve both the sensitivity and the selectivity of a cantilever by chemically modifying its surface.
A $\mathrm{TiO}_{2}$ nanostructure having high specific surface area was grown on cantilever for increasing its active surface. This concept has been highlighted in the work described by Spitzer and al., where $\mathrm{TiO}_{2}$ nanotubes prepared using anodization method were employed to increase the sensitivity of the cantilever and resulted in a detection limit of $0.8 \mathrm{ppb}$ of trinitrotoluene vapors [11]. In this work, such nanostructure was grown on the cantilever using a versatile solvothermal route, easier to achieve on small substrate than the anodization. In addition, the ability of the nanostructure to interact with chemicals was further improved by grafting specific molecules that selectively bind OPs. The detection tests were carried on a common OPs simulant, the DiMethylMethylphosphonate (DMMP).

\section{EXPERIMENTAL PROCEDURE}

\section{A. Wafer nanostructuration}

The nanostructure was initially achieved on a model surface (silicon wafer), and the optimized synthesis parameters were applied to the cantilevers surfaces. Before solvothermal synthesis, a thin layer of metallic titanium $(\sim 50 \mathrm{~nm})$ was first deposited on silicon surface by magnetron sputtering at a DC power density of $1.37 \mathrm{~W} . \mathrm{cm}^{-2}$ in order to compensate the lattice mismatch between silicon and $\mathrm{TiO}_{2}$. This layer was finally annealed at $800{ }^{\circ} \mathrm{C}$ during $6 \mathrm{~h}$ to oxidize the metallic film for improving the adherence and the growth of $\mathrm{TiO}_{2}$ nanostructure at the surface. The growth solution containing a mixture of 15 $\mathrm{ml}$ hydrochloric acid (37\%), absolute ethanol $(15 \mathrm{ml})$ and 0.25 $\mathrm{ml}$ of titanium isopropoxide ( $97 \%$ ) is prepared and loaded in the Teflon liner of autoclave. Afterwards, the coated substrate was dipped in the solution and the solvothermal process was done at $150{ }^{\circ} \mathrm{C}$ during 8 hours. The obtained sample was thoroughly washed with distilled water and dried under nitrogen flow. All the samples were characterized by x-ray diffraction (XRD) and scanning electron microscopy (SEM).

\section{B. Functionalization of the nanostructure}

A bifunctional molecule called (E)-4-((hydroxyimino) methyl)-benzoic acid is used to functionalize $\mathrm{TiO}_{2}$ nanostructure. The grafting mechanism involves a reaction between the hydroxyl function present at titanium dioxide 
surface and the carboxylic group of the molecule. The other part of the molecule contains an oxime function, well-known as nucleophile group that aid for the recognition of OPs molecules[10]. A mixture $(1: 1)$ of $5 \mathrm{ml}$ acetonitrile and tertbutanol is employed to dissolve $0.01 \mathrm{~g}$ of the molecule. The nanostructured silicon was immersed in the obtained solution and heat up for 24 hours at $70^{\circ} \mathrm{C}$. After the reaction, the sample was rinsed and analyzed by Raman and UV spectroscopy.

\section{Detection measurements}

The vapor generation system is composed of a DMMP saturator which delivers a precise concentration of the nerve simulant vapor (500 ppm). This vapor is then carried by a stream of air and sent to the detection chamber where the cantilever is located. Tipless silicon microcantilevers (TL-NCL-50 type) having $7 \mu \mathrm{m}$ of thickness, $225 \mu \mathrm{m}$ length, $38 \mu \mathrm{m}$ width and a nominal resonant frequency range of $146-236 \mathrm{kHz}$ were purchased from NanoSensors. The cantilever covered with the nanostructure is mounted in the head of a PicoSPM AFMmultimode to track the resonance frequency during the detection tests.

\section{RESULTS}

\section{A. $\mathrm{TiO}_{2}$ synthesis on wafer}

The diffractogram of oxidized titanium seed layer depicted on figure 1a showed the presence of pure tetragonal rutile phase of $\mathrm{TiO}_{2}$. This layer provides initial nucleation sites for growing a rutile nanostructure. On the same figure, we can observe the XRD pattern of the nanostructure film obtained after the solvothermal reaction. A single intense peak localized at $62.7^{\circ}$ is evidenced on the pattern, as expected; this peak is attributed to rutile phase. The absence of others main rutile peaks indicates that the nanostructure is highly oriented and has a preferred growth direction along the $c$ axis. Silicon diffraction peaks arise from the substrate can be also seen on this figure.

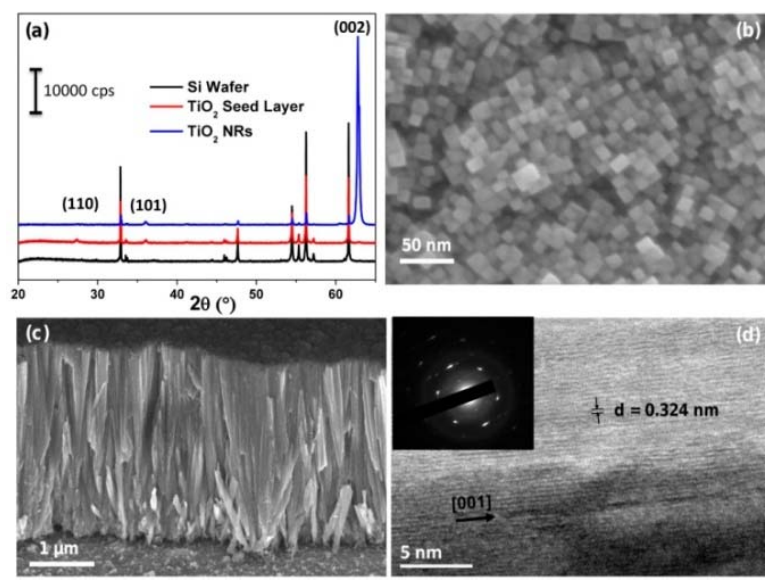

Figure 1: (a) XRD patterns of different films (b and c) SEM images of $\mathrm{TiO}_{2}$ nanorods grown on silicon wafer by solvothermal method (d) TEM image of a single rutile $\mathrm{TiO}_{2}$ nanorod.

The morphology of the nanostructure is exhibited on the SEM image reported on figure $1 b$ and c. The rutile $\mathrm{TiO}_{2}$ nanostructure which composed the film is highly dense and revealed vertically aligned nanorods with top square facet, commonly observed in tetragonal crystal structure. The nanorods are uniformly distributed on the Si surface, with an average length of $2.5 \mu \mathrm{m}$ and a width around $12 \mathrm{~nm}$. The preferential orientation of the nanorods is due to the adsorption of chloride ions on the lateral facets (110) of rutile crystal that hinders their growth and promotes the anisotropic growth of the nanostructure [12]. The monocrystalline structure of the film was also confirmed by the presence of few diffractions spots shown on the selected area diffraction pattern. Moreover, the measured interplanar distance $\left(\mathrm{d}_{110}=0.324 \mathrm{~nm}\right)$ using TEM image (Figure 1d) is in good agreement with the standard space between two (110) plans of rutile crystal [13].

\section{B. $\mathrm{TiO}_{2}$ functionalization}

We functionalized the as-synthesized nanorods surface with oxime molecules and its loading ability was estimated.

Figure 2a shows the Raman spectra of the reference nanostructure (Rut-EtOH) and the functionalized one (RutEtOH-Oxime). It can be found on both spectra, peaks at 443 and $609 \mathrm{~cm}^{-1}$ corresponding to $\mathrm{E}_{\mathrm{g}}$ and $\mathrm{A}_{1 \mathrm{~g}}$ active modes of rutile $\mathrm{TiO}_{2}$ polymorph respectively [14]. This result is in accordance with that observed in XRD analysis where the presence of rutile $\mathrm{TiO}_{2}$ phase has been already revealed. The intense narrow peak at 520 $\mathrm{cm}^{-1}$ and the large one peak at $943 \mathrm{~cm}^{-1}$ arise from silicon substrate [15]. In addition, for the grafted sample, two supplementary peaks located at 1412 and $1610 \mathrm{~cm}^{-1}$ can be observed. These peaks are typical vibration bands of COO group and $\mathrm{C}=\mathrm{C}$ from the aromatic ring[16], thereby suggesting the presence of oxime molecule onto rutile $\mathrm{TiO}_{2}$ nanorods surface of this sample. The grafted nanostructure was then immersed in $\mathrm{NaOH}$ solution at $\mathrm{pH}=12$, to desorb the molecules from the surface for UV visible spectroscopy quantification. The absorbance of the pi-pi* transition of the phenyl group (from oxime molecule) desorbed in solution was followed by UV spectroscopy to evaluate the amount of grafted oxime on the nanostructure surface. Using a calibration curve obtained via oxime titration, the estimated molecule density at the nanorods surface was $c a$. $70 \mathrm{nmol} . \mathrm{cm}^{-2}$.

\section{Effect of $\mathrm{TiO}_{2}$ synthesis solvent}

In order to find the best type of nanostructuration for chemical functionalization, various synthesis parameters were adjusted to control the morphology, the width and the length of the nanorods. We describe the effect of one of the most influential parameters (the solvothermal solvent).

The ethanol was replaced by ethylene glycol (EG) or water during the preparation of the growth solution. The SEM images of these samples (Figure 2b, c), showed that the morphology of rutile $\mathrm{TiO}_{2}$ phase obtained with water and EG differs significantly from the one prepared in ethanol medium. In the case of EG, well separated vertically oriented needles-like structure was obtained instead of the $\mathrm{TiO}_{2} \mathrm{NRs}$ with square facets given by ethanol. 

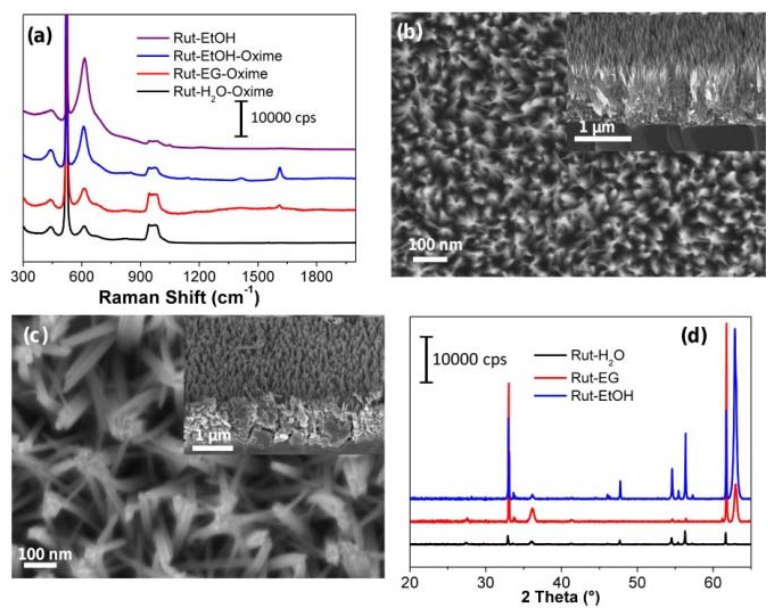

Figure 2: (a) Raman spectra of rutile nanorods and oxime-functionalized nanorods. SEM images of rutile prepared by changing ethanol with (b) Ethylene glycol and (c) Water (d) XRD of the nanostructure synthesized in water, glycol ethylene and ethanol.

In hydrothermal conditions, randomly oriented nanorods involving low density of nanorods at silicon surface was observed. Nevertheless, the rods-like structure which composed this new nanoarchitecture are agglomerated and should result in lower active surface for grafting. The XRD peaks of both films still indicate the presence of rutile phase. However, the intensity of resulting XRD peaks denotes that the orientation of the nanorods is strongly dependent on the nature of solvent. The needles structure morphology achieved with EG are less oriented compared to the nanorods with square facets. After performing the functionalization, the Raman spectra of the nanostructure synthesized in EG exhibited the two peaks related to the oxime molecule while they are not observed for the one prepared in water (Figure 2a). The number of adsorbed molecules estimated for the needles structure obtained in EG solvent is $34 \mathrm{nmol} . \mathrm{cm}^{-2}$, which corresponds to the half of the oxime density of the nanostructure obtained in ethanol (70 nmol. $\mathrm{cm}^{-2}$ ). As expected for the $\mathrm{TiO}_{2} \mathrm{NRs}$ synthesized in water, the molecules density is only $8 \mathrm{nmol} . \mathrm{cm}^{-2}$ due to the lower surface area.

\section{Sensor surface modification and detection tests}

Since $\mathrm{TiO}_{2}$ NRs synthesis in ethanol gives the nanostructure with the higher adsorption capacity, we applied this optimized synthesis for cantilevers nanostructuration. From the SEM image of this sample depicted on Figure 3a, we can see that a homogeneous layer of $\mathrm{TiO}_{2}$ nanostructure completely covers the surface of the microcantilever. The morphology of $\mathrm{TiO}_{2}$ obtained at this micrometric surface is very similar to $\mathrm{TiO}_{2}$ nanostructure synthesized on centimetric silicon wafers. The growth of similar nanostructure on both types of support highlighted the reproducibility and the versatility of this optimized $\mathrm{TiO}_{2}$ NRs synthesis. The modified cantilever was tested for sensing DMMP vapor. For comparison, the detection tests were also performed with cantilevers nanostructured in the others solvents (EG and water). Figure $3 \mathrm{~b}$ shows the evolution of the resonance frequency over the time of pristine cantilever and cantilevers covered with different nanostructure.

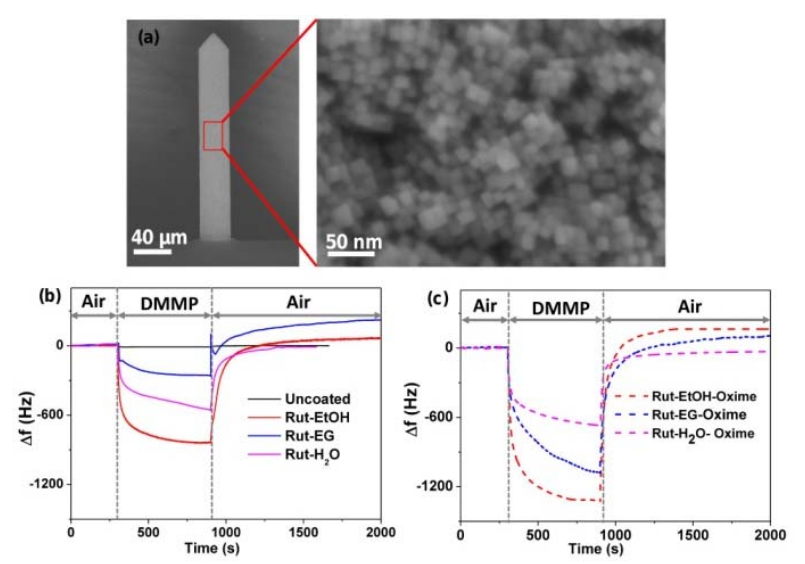

Figure 3 : (a) $\mathrm{TiO}_{2}$ nanostructured cantilever prepared in ethanol. Measured sensing frequency responses to $500 \mathrm{ppm}$ of DMMP vapor of (b) pristine microcantilever and the one prepared in different solvents (c) oxime functionalized cantilevers.

All the tested sensors showed a resonance frequency drop when $500 \mathrm{ppm}$ of DMMP vapors was generated near their surfaces within a few seconds. The frequency shifts correspond to $11,256,556$ and $833 \mathrm{~Hz}$ for uncoated microcantilever and the ones nanostructured in ethylene glycol, water and ethanol respectively. These results indicate the adsorption of DMMP on the cantilevers surfaces and therefore the detection of the target molecule. When the air containing the DMMP vapor is replaced after 10 minutes by the reference air flux, the DMMP molecules are desorbed from the nanostructured cantilevers, and the resonance frequency returns to the baseline value after a short period. A cycle measurement of each modified sensor was performed and resulted in a good repeatability.

The higher frequency shift obtained with the cantilever nanostructured in ethanol, confirms that a large part of its surface area is accessible for the analyte even though the film appears highly dense. The same sensor exhibits the highest frequency shift of $1319 \mathrm{~Hz}$ when the oxime molecules are adsorbed on its surface, as expected for the high surface area and the high loading of grafting offered by the nanostructure (Figure 3c). A theoretical detection limit of $2.25 \mathrm{ppm}$ of DMMP with this sensor is obtained. Although this concentration remains high compared to the OPs detection limits (5 and 7.2 ppb) reported for some gravimetric sensors [17], [18], this result provides a kind of guideline for developing new nanostructures with higher sensitivity.

\section{CONCLUSION}

The synthesis of $\mathrm{TiO}_{2}$ NRs on microcantilever surface was demonstrated using a solvothermal method. The control of the morphology of $\mathrm{TiO}_{2}$ was also evidenced and the results showed that the surface area developed by the nanostructure has a high impact on the oxime loading. The proposed approach to increase the active surface of cantilever was very beneficial to significantly improve by a factor of 75 the sensitivity of the cantilever for sensing OPs compounds. We also found that the presence of grafted molecules at the surface almost doubles the response of the nanostructured cantilever even though oxime was only aimed to enhance sensor selectivity. 


\section{REFERENCES}

[1] M. Alvarez and L. M. Lechuga, "Microcantilever-based platforms as biosensing tools," Analyst, vol. 135, no. 5, pp. 827-836, Apr. 2010.

[2] Chaudhary, Monika, Gupta, and Amita, "Microcantilever-based Sensors.," Defence Science Journal, vol. 59, no. 6, pp. 634-641, 2009.

[3] K. M. Hansen and T. Thundat, "Microcantilever biosensors," Methods, vol. 37, no. 1, pp. 57-64, Sep. 2005.

[4] M. Calleja, M. Nordström, M. Álvarez, J. Tamayo, L. M. Lechuga, and A. Boisen, "Highly sensitive polymer-based cantilever-sensors for DNA detection," Ultramicroscopy, vol. 105, no. 1, pp. 215-222, Nov. 2005.

[5] T. Thundat, G. Y. Chen, R. J. Warmack, D. P. Allison, and E. A. Wachter, "Vapor Detection Using Resonating Microcantilevers," Anal. Chem., vol. 67, no. 3, pp. 519-521, Feb. 1995.

[6] L. A. Pinnaduwage, V. Boiadjiev, J. E. Hawk, and T. Thundat, "Sensitive detection of plastic explosives with self-assembled monolayer-coated microcantilevers," Appl. Phys. Lett., vol. 83, no. 7, pp. 1471-1473, Aug. 2003.

[7] D. Lee, O. Zandieh, S. Kim, S. Jeon, and T. Thundat, "Sensitive and selective detection of hydrocarbon/water vapor mixtures with a nanoporous silicon microcantilever," Sens. Actuators B Chem., vol. 206, pp. 84-89, Jan. 2015.

[8] Y. Yang, H.-F. Ji, and T. Thundat, "Nerve Agents Detection Using a Cu2+/l-Cysteine Bilayer-Coated Microcantilever," J. Am. Chem. Soc., vol. 125 , no. 5, pp. 1124-1125, Feb. 2003.

[9] R. K. Ahmad, A. C. Parada, S. Hudziak, A. Chaudhary, and R. B. Jackman, "Nanodiamond-coated silicon cantilever array for chemical sensing," Appl. Phys. Lett., vol. 97, no. 9, p. 93103, Aug. 2010.

[10] J. Kassa, "Review of oximes in the antidotal treatment of poisoning by organophosphorus nerve agents," J. Toxicol. Clin. Toxicol., vol. 40, no. 6, pp. 803-816, 2002.

[11] D. Spitzer et al., "Bio-Inspired Nanostructured Sensor for the Detection of Ultralow Concentrations of Explosives," Angew. Chem. Int. Ed., vol. 51, no. 22, pp. 5334-5338, 2012.

[12] D.-D. Qin et al., "Hydrothermal Growth and Photoelectrochemistry of Highly Oriented, Crystalline Anatase TiO2 Nanorods on Transparent Conducting Electrodes," Chem. Mater., vol. 27, no. 12, pp. 4180-4183, juin 2015.

[13] A. Kumar, A. R. Madaria, and C. Zhou, "Growth of Aligned SingleCrystalline Rutile TiO2 Nanowires on Arbitrary Substrates and Their Application in Dye-Sensitized Solar Cells," J. Phys. Chem. C, vol. 114, no. 17, pp. 7787-7792, May 2010.

[14] V. V. Burungale, V. V. Satale, A. M. Teli, A. S. Kamble, J. H. Kim, and P. S. Patil, "Surfactant free single step synthesis of TiO2 3-D microflowers by hydrothermal route and its photoelectrochemical characterizations," J. Alloys Compd., vol. 656, pp. 491-499, Jan. 2016.

[15] B. Li, D. Yu, and S.-L. Zhang, "Raman spectral study of silicon nanowires," Phys. Rev. B, vol. 59, no. 3, pp. 1645-1648, Jan. 1999.

[16] C. Pérez León, L. Kador, B. Peng, and M. Thelakkat, "Characterization of the Adsorption of Ru-bpy Dyes on Mesoporous TiO2 Films with UV-Vis, Raman, and FTIR Spectroscopies," J. Phys. Chem. B, vol. 110, no. 17, pp. 8723-8730, May 2006.

[17] S. Cai et al., "In situ construction of metal-organic framework (MOF) UiO-66 film on Parylene-patterned resonant microcantilever for trace organophosphorus molecules detection," Analyst, vol. 144, no. 12, pp. 3729-3735, Jun. 2019.

[18] Y. Liu et al., "Hyper-branched sensing polymer directly constructed on a resonant micro-cantilever for the detection of trace chemical vapor," J. Mater. Chem., vol. 22, no. 34, pp. 18004-18009, Aug. 2012. 